\title{
BEA. La capilla submarina viajera propuesta por Mark Mills para la liturgia postconciliar americana
}

BEA. The traveling underwater chapel proposed by Mark Mills for American post-conciliar liturgy

Nuria Prieto González · Universidade da Coruña (España)

https://doi.org/10.17979/aarc.2013.3.0.5089

\section{RESUMEN}

En 1970, el padre Joseph Champlin escribe: «la vasta mayoría espera esta nueva época en la vida de nuestra Iglesia». Mark Mills realiza en 1971 el proyecto BEA, una capilla submarina inspirada en la forma de un pez manta que viajaría alrededor del mundo constituyéndose como un «símbolo marino dedicado a la paz». La propuesta, vista como un proyecto realista, sigue la línea de las arquitecturas contraculturales paralelas a Fuller, en las que el hábitat del hombre y su relación con la naturaleza y la comunidad se convierten en elementos esenciales del proyecto, definiendo más un espacio cognitivo o sensorial que simbólico o figurativo.

\section{ABSTRACT}

In 1970 father Joseph Champlin writes: «the vast majority waits this new epoch in the life of our church». Mark Mills develops in 1971 the project BEA, a submarine chapel inspired in the form of a manta ray, that would travel all over the world becoming a «marine symbol consecrated to peace». The proposal, seen as a realistic project, follows the research of countercultural architectures next to Fuller's, in which the habitat of man and his relationship with nature and the community become essential elements of the project, defining a more cognitive and sensible space but symbolic or figurative.

\section{PALABRAS CLAVE}

Contracultura; capilla; Mark Mills; arquitectura móvil; ecuménico.

\section{KEYWORDS}

Counterculture; chapel; Mark Mills; mobile architecture; ecumenical. 
Fig. 01. Martin Pulaski, Sin título, 1968.

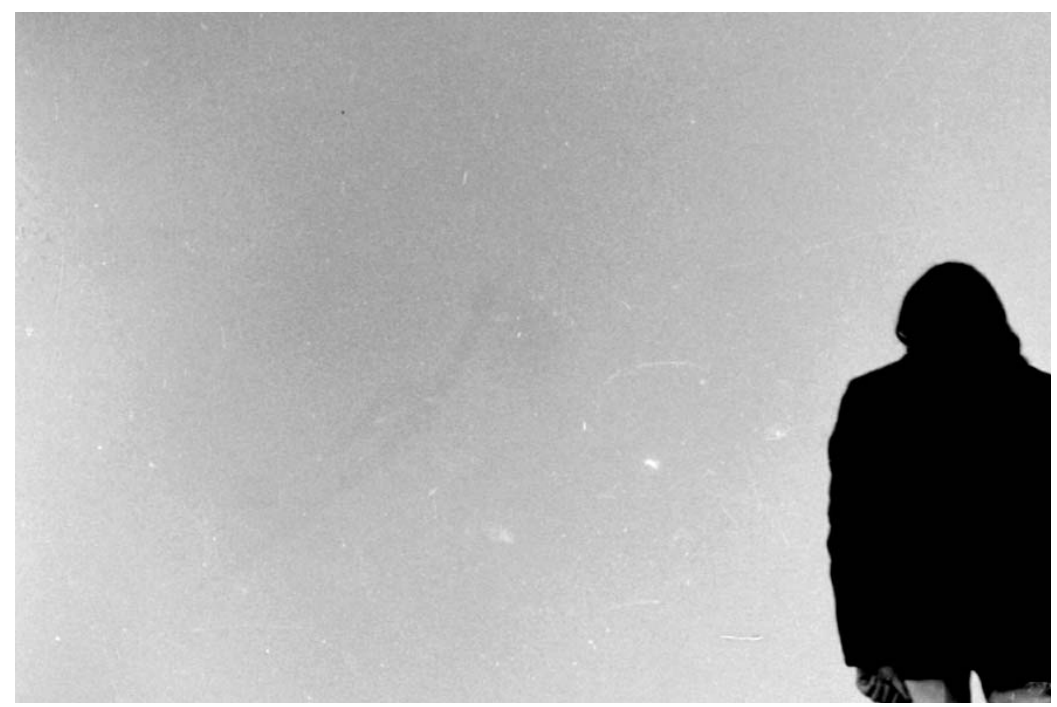

La década de los setenta es, para la sociedad contemporánea, la década de la revolución cultural. Desde EEUU llegaban los ecos de un cambio social entre los jóvenes, una ruptura con el tradicionalismo cultural que alcanzaría a todos los niveles de la sociedad. El nacimiento de la contracultura es el producto de una nueva perspectiva, que aplicada a la arquitectura, desbordará sus límites, quebrando en muchas ocasiones conceptos tradicionales o académicos.

El lenguaje arquitectónico asimila el cambio y aparecen conceptos como drop out, trip o hip-type ${ }^{1}$, una terminología que será necesaria para explicar la mayor parte de las propuestas que se enmarcan dentro de esta década. El límite de la arquitectura es sometido a crítica, definiendo un ámbito de producción difuso que traza una línea discontinua englobando la instalación artística, el vídeo, los manifiestos o las construcciones efímeras. En el Manifiesto de la Ciudad Instantánea de Ibiza (1971) se expresaba claramente este nuevo camino: «El mundo se está preparando para una metamorfosis de los dioses. Se abandonan los valores y arquetipos de la cultura vigente y se adoptan nuevas formas de vida, nacidas de otra visión del mundo. Uno de los valores de la Nueva Cultura es el propio cambio, la impermanencia, la flexibilidad $»^{2}$. Estas palabras, inspiradas en los movimientos de la contracultura americana, presentan un cierto paralelismo con las del padre Joseph Champlin, quien en 1970 había escrito: «la vasta mayoría espera esta nueva época en la vida de nuestra Iglesia» ${ }^{3}$. Un cambio: un cambio optimista, heredero de los valores alegres de la década de los sesenta y que pretende ser la resultante positiva de una etapa convulsa (Fig. 01).

El optimismo de la nueva liturgia derivada del Concilio Vaticano II se materializa en el comentario que el padre Joseph Champlin realiza sobre la GIRM de $1970^{4}$, resumiendo brevemente los puntos más interesantes de ésta. Champlin es consciente del cambio al que se enfrenta la comunidad católica estadounidense, que se ve sumida en los movimientos sociales a favor de los Derechos Civiles, así como en la lucha por los derechos de la mujer, y que se encontraba gravemente afectada por el declive de la propia entidad, que se reflejaba en la deserción de algunos párrocos, el cierre de instituciones y la polémica sobre la enseñanza católica en los centros públicos. Según recoge Champlin resumidamente, la nueva Instrucción planteaba: la flexibilización de algunas normas; la participación personal en la liturgia, de forma que el individuo se sienta más integrado en la comunidad; la focalización de la misma liturgia en la comunidad; la variedad y el dinamismo en los actos religiosos, transformándolos en una actividad atractiva; la calidad de estas ceremonias, de forma que incluyan música, lecturas e incluso la renovación del mobiliario; y finalmente, un énfasis en la 
Fig. 02. Reunión de jóvenes católicos en el Mother Centre, Nambassa Winter Show (Nueva Zelanda), 1976.

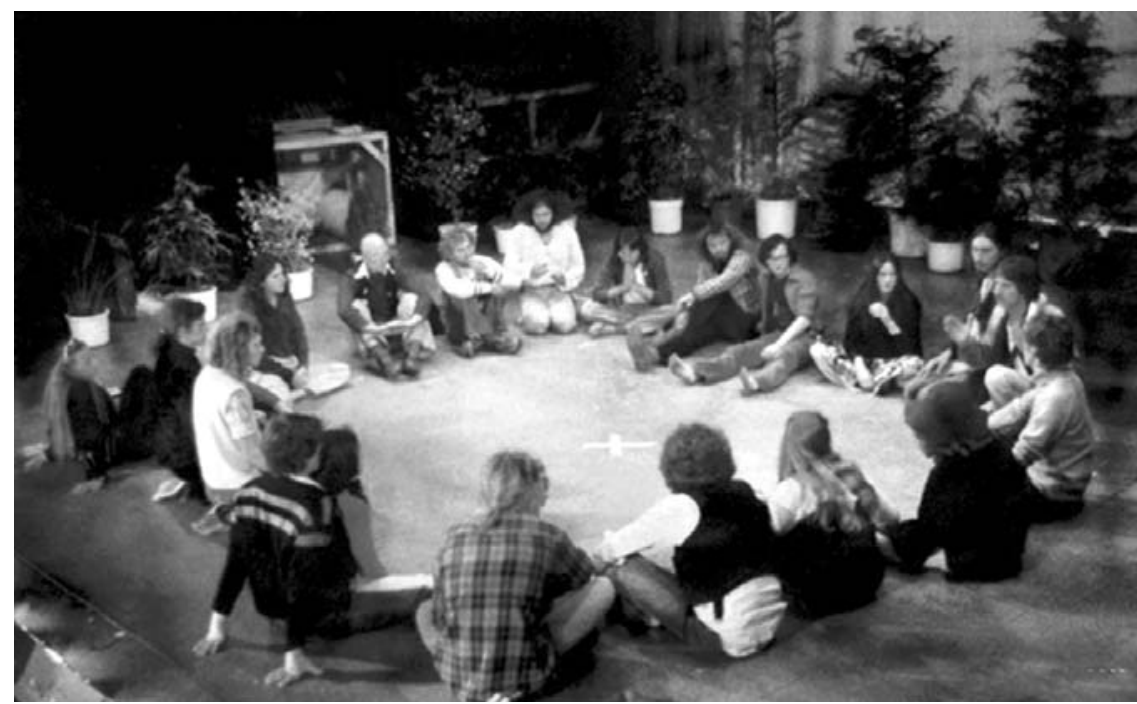

claridad del mensaje. Esta última característica es especialmente subrayada por el Concilio Vaticano II como premisa importante para la renovación de la Iglesia (Fig. 02). La GIRM de 1970 se convierte en un manifiesto que puede adjetivarse como contracultural dentro de la estructura eclesiástica norteamericana de entonces. Por esta razón, Champlin ve en este documento un motivo de optimismo que modernizará la imagen de la Iglesia católica en EEUU. Una mirada que introduce a la institución dentro del hip-type formulado por Hedgepeth en 1966.

La convulsa situación histórica que atraviesan los EEUU en la década de los setenta tiene como protagonista la guerra de Vietnam. Ésta se convierte en el objeto de reclamo que ejemplifica las luchas por los derechos civiles, la paz y las libertades. La guerra de Vietnam servirá también de inspiración del proyecto titulado «BEA. La capilla submarina viajera» del arquitecto Mark Mills, un proyecto que refleja los principios contraculturales, así como los aspectos más importantes de la GIRM de 1970. Este proyecto tan singular permite un análisis sinóptico, contextualizando la capilla en la trayectoria profesional de Mills y posicionándola dentro del grupo de propuestas arquitectónicas contraculturales de la década de los setenta.

Mark Mills fue uno de los muchos arquitectos recién graduados que pasó bastante tiempo trabajando en Taliesin con Frank Lloyd Wright. Su estancia sería de cuatro años (entre 1944 y 1948), abandonando el estudio con Paolo Soleri, quien sería su socio en el primer proyecto fuera del Taliesin: la Cave Creek en Arizona. La arquitectura de Mills es caracterizada por sus contemporáneos como orgánica, ya que utiliza formas que manifiestan un paralelismo directo con estructuras biológicas. Dentro de la producción de Mills que se encuentra en su mayoría en el estado de California- destacan algunas obras como la Fan Shell House o la Cooper Spine House ${ }^{5}$.

La revista Liturgical Arts recoge en su número 40 (noviembre de 1971) el proyecto BEA de Mark Mills. Este proyecto supone una más de las múltiples propuestas que en la década de los setenta que se enumeran bajo el grito habiter la mer. Propuestas que no son ajenas al lugar, sino que lo perciben en global, aplicando una de las máximas de Buckminster Fuller: «We are all astronauts» ${ }^{6}$. Norberg-Schultz explicaba claramente en 1970 que «el lugar en la cultura contemporánea se estaba comenzando a disolver, y que este proceso se manifestaba tanto en la extensa percepción social como en la experiencia de ser desenraizado de la insuficiencia figurativa del planeamiento contemporáneo»?

La definición de una ciudad flotante se convierte en obsesión para muchos arquitectos como Frank Lloyd Wright, Paolo Soleri, William Katavolos, 

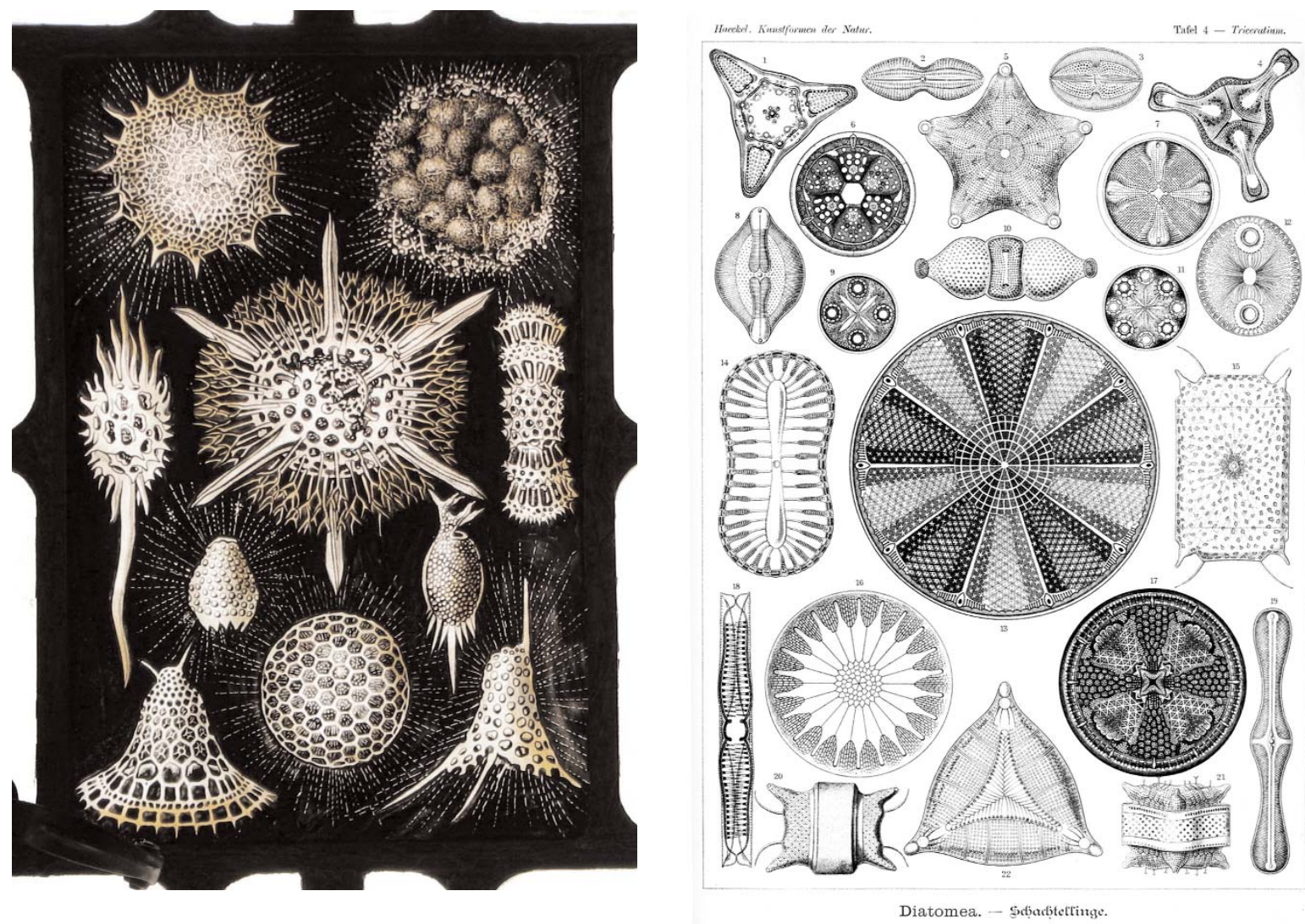

Fig. 03. Radiolarios de Haeckel sobre vidrio, 2010. Fig. 04. Radiolarios de Haeckel, 2009.

Fig. 05. Pez manta, 2011.

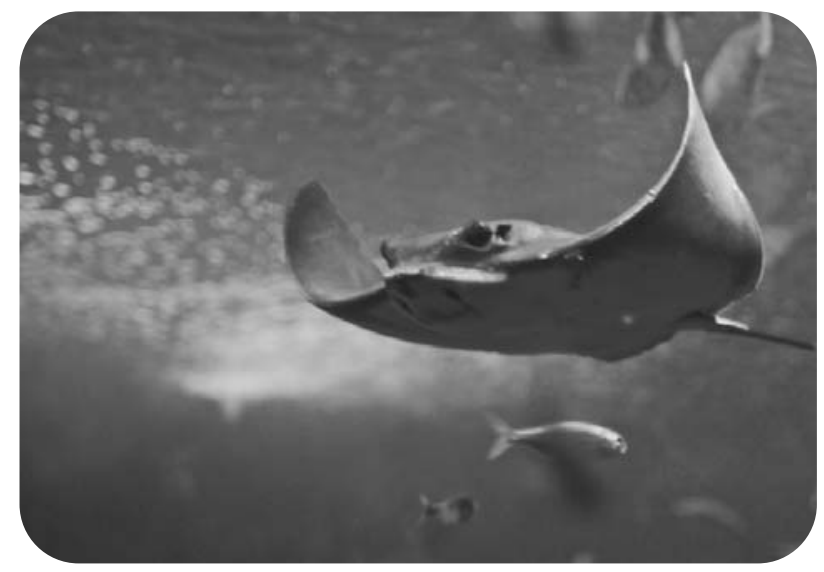




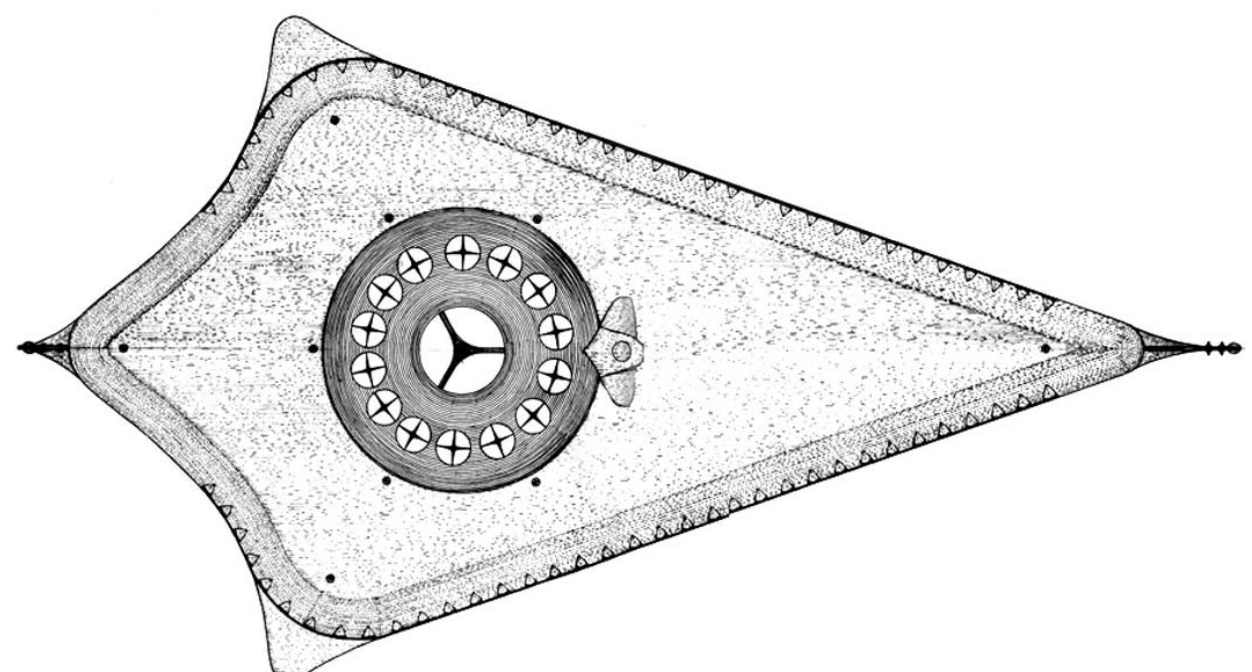

Fig. 06. Mark Mills, Submarine for Liturgical Arts Society, 1971; planta.

Noriaki Kurokawa o Buckminster Fuller. Sus propuestas conceptuales describen unas estructuras de carácter esencialmente orgánico que, o bien flotan o bien se sumergen en el mar, pero siempre teniendo en cuenta que «las estructuras construidas han de poder ser, en caso de necesidad extrema, destruidas, desplazadas o desmontadas», de manera que así «no se destruya el equilibrio de la evolución de los ecosistemas de la zona utilizada» ${ }^{8}$. Esta última cita de Jacques Rougerie es clave para determinar las características constructivas y estructurales de las propuestas en apariencia utópicas.

La morfología estructural de las propuestas para habitar el mar se apoyan en dibujos o radiolarios de fósiles animales, como los de Haeckel, que estudiaba el ingeniero francés Robert Le Ricolais (Fig. 03-04)9. Los trabajos de Le Ricolais sobre las morfologías orgánicas eran compartidos por Buckminster Fuller y Frei Otto ${ }^{10}$, quienes solían discutir amistosamente sobre ellos. Miller, por su parte, plantea un proyecto heredero de esas investigaciones, ya que su capilla pretende ser un animal que recorre el océano.

Para Miller y para los editores de la revista en la que aparece publicada por primera vez, «esta capilla submarina, consagrada únicamente a la paz (...) es un sueño posible» ${ }^{11}$. Fusionando en el mismo proyecto las aspiraciones pacifistas de aquéllos que rechazaban la traumática guerra de Vietnam con la idea de una arquitectura orgánica viajera submarina — tan estudiada por sus contemporáneos-, Miller crea esta capilla con una misión: recorrer el mundo buscando la paz y la armonía entre los pueblos. En resumen: «Una expresión tangible del bien hacia el hombre, que viaja alrededor del mundo» ${ }^{12}$. Este espíritu misionero se plasmará, no sólo en el plan de viaje de la capilla — que realizará rutas de cinco años de duración-, sino también en su nombre, $B e a$, en honor al cardenal jesuita Augustin Bea (1881/1968) y al barco de investigación de Charles Darwin, el Beagle.

La forma de la capilla es la de un pez manta (Fig. 05), que para Mills era la forma más efectiva: la que emulaba a un tiburón y era la utilizada por los submarinos militares. Se compone de dos partes: un cuerpo central de forma trapezoidal y proporciones alargadas, $\mathrm{y}$ otro volumen esférico que interseca al primero en su centro y del que salen óculos circulares (Fig. 06-07). La volumetría de la capilla es la de un animal de aspecto mitológico, en cuyo interior el espacio de carácter religioso busca ser multifuncional. Recuperando la interpretación que el padre Champlin hacía de la GIRM de 1970, Mills propone que el espacio central — de grandes dimensiones - pueda ser utilizado como teatro o como centro de investigación submarina cuando no se utilice como capilla. Formula así un espacio de convi- 


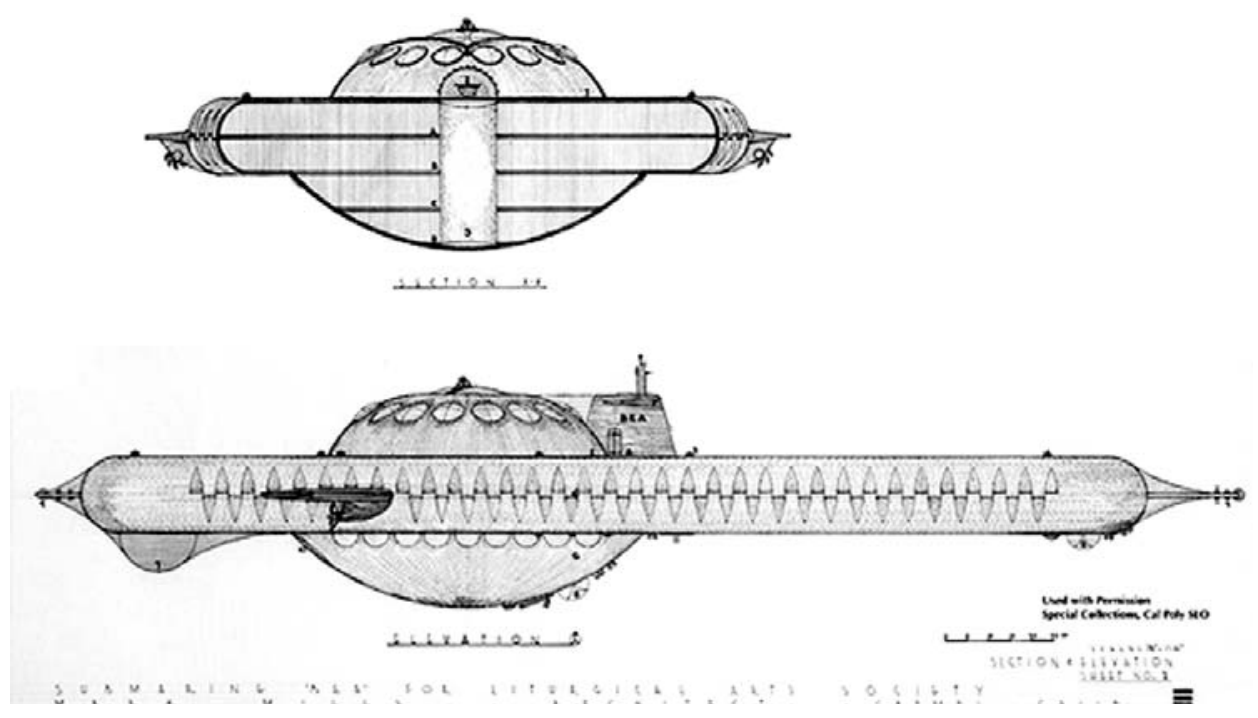

Fig. 07. Secciones.

vencia, en el que el fin común sea - como se dice en la memoria- «honrar a Dios a través de la variedad de fes y liturgias» ${ }^{13}$. El espacio esférico secundario permite crear pequeñas capillas y espacios de reflexión, que cuando no sean utilizados como tales puedan ser observatorios del fondo marino.

Aunque inicialmente la capilla se plantea de confesión católica, su voluntad viajera, misionera y pacifista la convierte en un espacio ecuménico, en que cualquier ser humano, de cualquier raza o condición, pueda encontrarse en comunidad y armonía a través de la espiritualidad. Ese estar en medio de la nada, en la profundidad del océano, convierte a esta capilla en un lugar de reflexión único. En palabras de Alvar Aalto, una «arquitectura que tiene un motivo más allá, la idea de crear el paraíso. Deseamos crear el paraíso para el hombre en la tierra» ${ }^{14}$. Pero al mismo tiempo, esa relación directa con la naturaleza tan propia de las arquitecturas de la década de los setenta, se convierte en un motivo más de sacralidad. Como dice Pallasmaa, «la belleza natural, un paisaje en particular o incluso unas condiciones meteorológicas particulares a menudo despiertan los sentimientos de serenidad y sacralidad» ${ }^{15}$. La capilla es así un espacio cognitivo ${ }^{16}$ (Fig. 08), acentuado por las múltiples posibilidades que se plantean desde el proyecto, como la proyección de imágenes o luces, o la introducción de sonidos marinos reales.
La búsqueda de una conexión tan directa con la naturaleza es también una vuelta al origen. Un paralelismo gaudiniano en la formalización de un espacio orgánico y original que, «sea cual sea el nombre que le demos al sentimiento que produce experimentar esta arquitectura, podemos reconocer que nuestra memoria encuentra inmediatamente las trazas de una felicidad primaria, un paraíso que no está enteramente perdido» ${ }^{17}$.

La capilla comenzaría su viaje en Alcatraz, San Francisco, recorrería el mundo con paradas especiales en lugares como Bikini o Nagasaki, y volvería a Filadelfia para la gran feria mundial de 1976 (Fig. 0910). Un largo viaje de cinco años al que cualquiera podría sumarse; una capilla misionera católica en busca de la paz mundial. Un planteamiento que no se aleja mucho del conocido World Game de Buckminster Fuller, cuyo objetivo era también la globalización desde un punto de vista pacifista ${ }^{18}$.

Bea, the submarine chapel, es un lugar de convivencia religiosa que pretende materializar las ideas de la GIRM 1970, así como manifestarse a favor de la paz mundial, la tolerancia y la convivencia a través de una nueva forma de comprender la liturgia renovada. Una perspectiva contracultural que permite entender la arquitectura de la Iglesia católica contemporánea más vanguardista. 


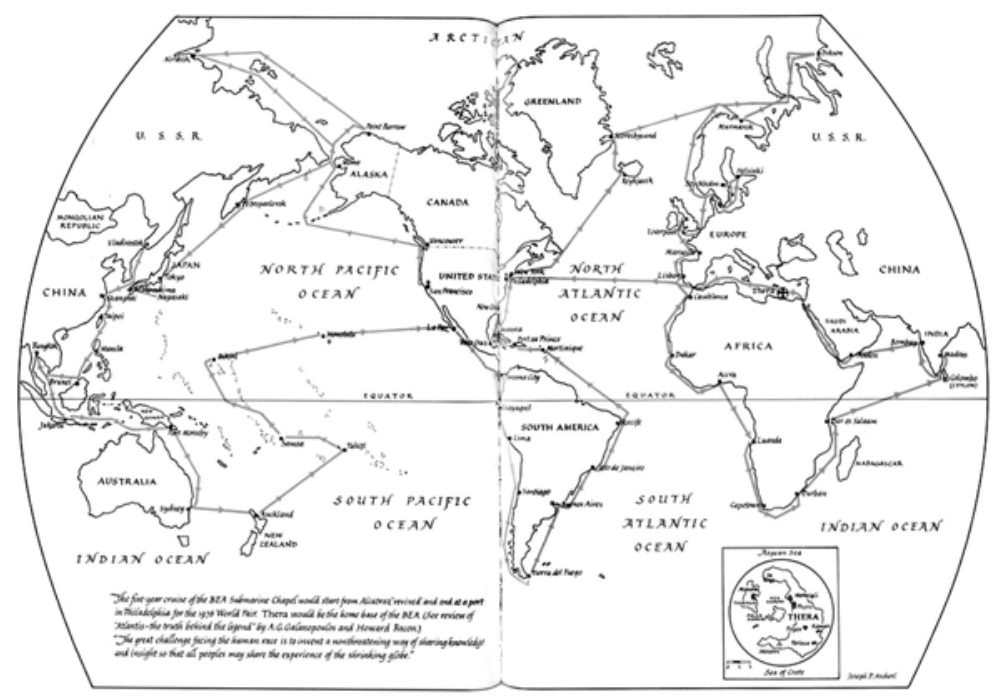

Fig. 08. We-make-money-not-art, Corazón Amarillo, 2011 (abajo). Fig. 09. Mark Mills, Submarine for Liturgical Arts Society, 1971; mapa de ruta (izquierda). Fig. 10. Llegada a puerto (en la página siguiente).

\section{CRÉDITOS DE LAS ILUSTRACIONES}

Fig. 01-04 y 07. Con acceso el 10/10/2013, www.flickr.com/

Fig. 02. Con acceso el 10/10/2013, http://en.wikipedia.org/ wiki/Nambassa_Winter_Show_with_Mahana.

Fig. 05-06 y 08-10. Con acceso el 13/06/2013, www.kcet.org/arts/artbound/counties/san-luis-obispo/coastalmodern-mark-mills-architect.html.

\section{NOTAS}

(1) Términos definidos por William Hedgepeth, considerado como el primer cronista del movimiento hippie y crítico de su arquitectura. Cf. Felicity D. Scott, Architecture or Techno-Utopia: Politics after modernism (Cambridge: MIT University Press, 2007), 163.

(2) Comité Ad Hoc de la Ciudad Instantánea, Manifiesto (Ibiza: Comité Ad Hoc, 1971), 1.

(3) Joseph Champlin, «A New Epoch in the Liturgy», Liturgical Arts 38 (1970): 82-83.

(4) General Instruction on the Roman Missal (GIRM), 1970. Con acceso el 13 de junio de 2013, www.ewtn.com/library/curia/girmall.htm.

(5) Texto recogido en www.janeybennett.com, originalmente publicado en Journal of the Taliesin Fellows 10 (1993): 18-29.

(6) Richard Buckminster Fuller \& Jaime Snyder, And they Came to pass not to Stay (Middlesex: Penguin Books Ltd., 2008).

(7) Cf. Maria Antonietta Crippa, «A Dwelling for Man within the Harmony of the Cosmos», en The religious imagination in modern and contemporary architecture: A Reader, ed. Renata Hejduk \& Jim Williamson (New York: Routledge Taylor and Francis Group, 2011), 104.
(8) Jacques Rougerie, «Urbanisme et architecture de la mer», Architecture d'aujourd'hui 175 (1974): 66-76.

(9) Robert Le Ricolais, Visiones y Paradojas (Madrid: COAM), 1997.

(10) Josep María Songel, Frei Otto (Barcelona: Artes Gráficas Campás, 1997).

(11) Joanne M. Pierce, «A Chapel on the Moon: Reflections on Roman Catholic Liturgical Imagination in 1967 and 2007», en Colloquium: Music, Worship, Arts 5 (2008): 11-26. También disponible en www.yale.edu/ism/colloq_journal/vol5/index.html.

(12) Pierce, «A Chapel on the Moon», 2.

(13) Ibídem, 4.

(14) Juhani Pallasmaa, «The Aura of the Sacred: Art, Architecture and Existential Sacredness», en Hejduk, The religious imagination, 234.

(15) Loc. cit.

(16) Esta característica era habitual en proyectos vanguardistas de la época como el mind expander, Corazón amarillo o Reservat, de Haus Rucker Co.

(17) Crippa, «A Dwelling for Man», 104.

(18) Fuller \& Snyder, And they Came, 35.

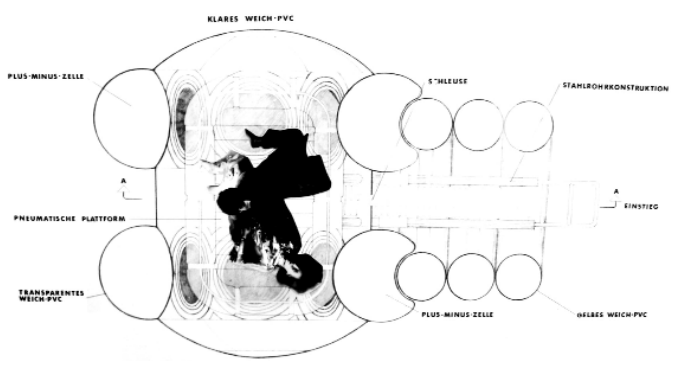




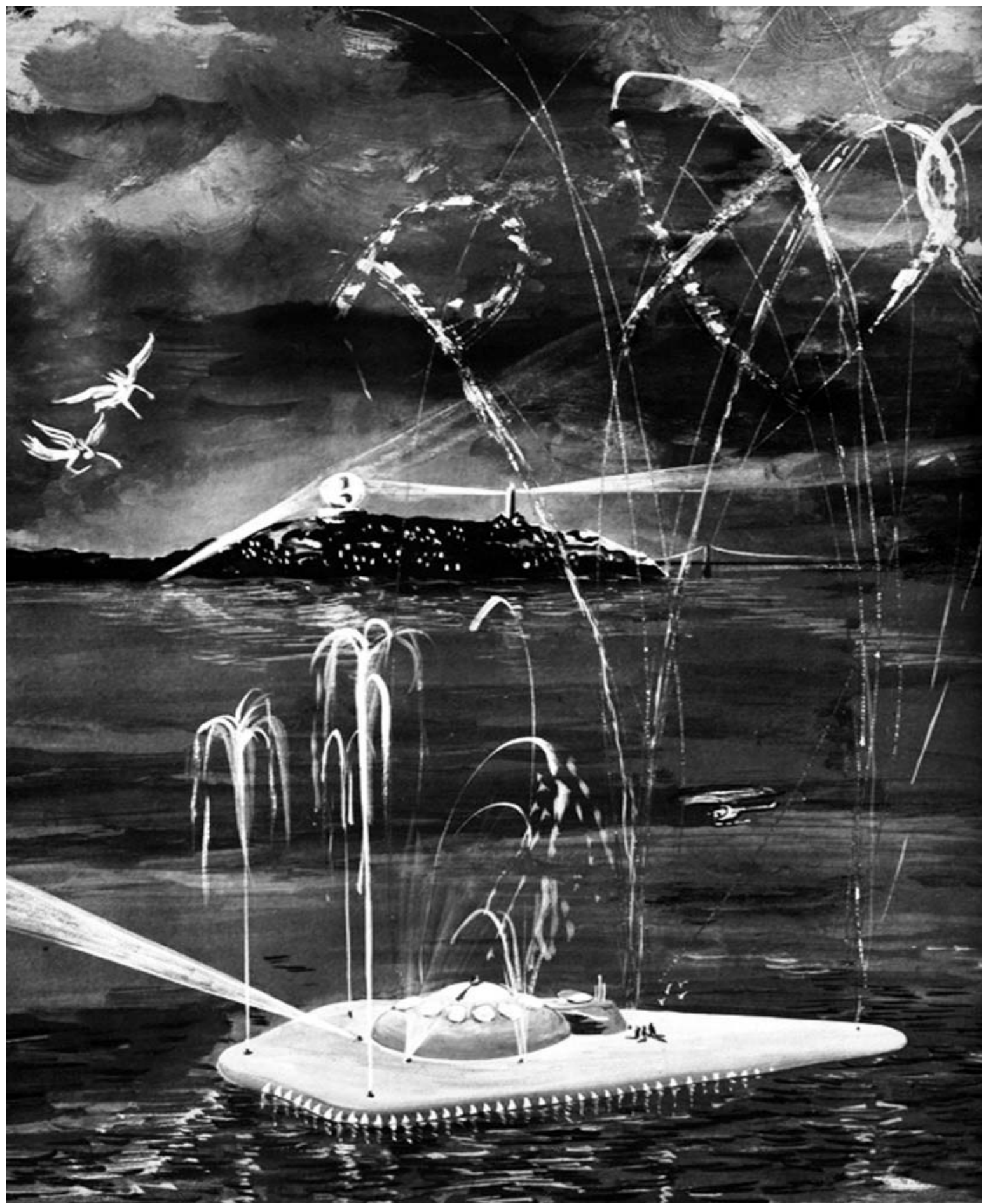

